\title{
Implications on the collision dynamics via azimuthal sensitive HBT from UrQMD
}

\author{
Gunnar Gräf* \\ Frankfurt Institute for Advanced Studies (FIAS), Germany \\ Institut für Theoretische Physik, Goethe Universität Frankfurt, Germany \\ E-mail: graef@th.physik. uni-frankfurt.de
}

\section{Elliot Mount}

Department of Physics, Ohio State University, Columbus, Ohio 43210, USA

\section{Michael Annan Lisa}

Department of Physics, Ohio State University, Columbus, Ohio 43210, USA

\section{Marcus Bleicher}

Frankfurt Institute for Advanced Studies (FIAS), Germany

Institut für Theoretische Physik, Goethe Universität Frankfurt, Germany

\begin{abstract}
We explore the shape and orientation of the freezeout region of non-central heavy ion collisions. For this we fit the freezeout distribution with a tilted ellipsoid. The resulting tilt angle is compared to the same tilt angle extracted via an azimuthally sensitive HBT analysis. This allows to access the tilt angle experimentally, which is not possible directly from the freezeout distribution. We also show a systematic study on the system decoupling time dependence on $d N_{c h} / d \eta$, using HBT results from the UrQMD transport model. In this study we found that the decoupling time scales with $\left(d N_{c h} / d \eta\right)^{1 / 3}$ within each energy, but the scaling is broken across energies.
\end{abstract}

The Seventh Workshop on Particle Correlations and Femtoscopy

September 20 - 242011

University of Tokyo, Japan

* Speaker. 


\section{Introduction}

In the early universe shortly after the big bang existed a strongly interacting state of matter called the quark-gluon plasma (QGP). The strong interaction is described by the theory of quantum chromodynamics (QCD). To investigate the details of QCD we recreate the conditions present in the early universe. Today these conditions can only be found in the interior of neutron stars or be created in heavy-ion collisions at relativistic energies. For probing the strongly interacting matter, various experiments are running at colliders, such as the SPS, RHIC and LHC. However in all of these experiments neither the extent of the particle emission region, nor the particle emission duration is directly accessible since the size is only on the order of a few fm. Nevertheless by using Hanbury Brown-Twiss (HBT) interferometry it is possible to extract the size and even the spatial orientation of the particle emission zone created in these collisions.

\section{Description of the UrQMD model and the Gaussian fitting procedure}

For the study of HBT correlations we employ the Ultrarelativistic Quantum Molecular Dynamics (UrQMD) [1, 2] in version 3.3 (for details of version 3.3 see [3, 4]). The source of the model is available at [5]. UrQMD is a hadronic non-equilibrium transport model which describes the whole spacetime evolution of nucleus-nucleus collisions from the beginning until the kinetic freezeout. Particles in UrQMD are produced via hard collisions, string excitation and fragmentation, and via resonance excitation and decay.

To extract the pion HBT radii we calculate the HBT correlation function in the longitudinal comoving system (LCMS) by [6]

$$
C(\mathbf{q}, \mathbf{K})=1+\int d^{4} x \cos (q \cdot x) d(x, K),
$$

where $C$ is the correlation function, $q$ is the four-momentum distance of the correlated particles, $K=\left(p_{1}+p_{2}\right) / 2$ is the pair momentum, $x$ is the particle separation four-vector and $d$ is the normalized pion freezeout separation distribution.

The HBT radii $R_{i j}$ are then obtained by fitting the function

$$
C(\mathbf{q}, \mathbf{K})=1+\lambda(\mathbf{K}) \exp \left[-\sum_{i, j=o, s, l} q_{i} q_{j} R_{i j}^{2}(\mathbf{K})\right]
$$

to the calculated three-dimensional correlation functions.

For the azimuthal sensitive analysis of the HBT correlations the space is subdivided in several azimuthal sections around the beam axis. For each of the sections an individual correlation function is computed. The azimuthal angle of the pair momentum vector determines in which correlation function each pion pair is counted.

\section{Azimuthally sensitive HBT from UrQMD generated correlation functions}

For the azimuthal analysis each of the correlation functions is fitted with Eq. 2.2 separately. The resulting HBT radii are plotted versus the corresponding azimuthal angle in Fig. 1. An obvious 

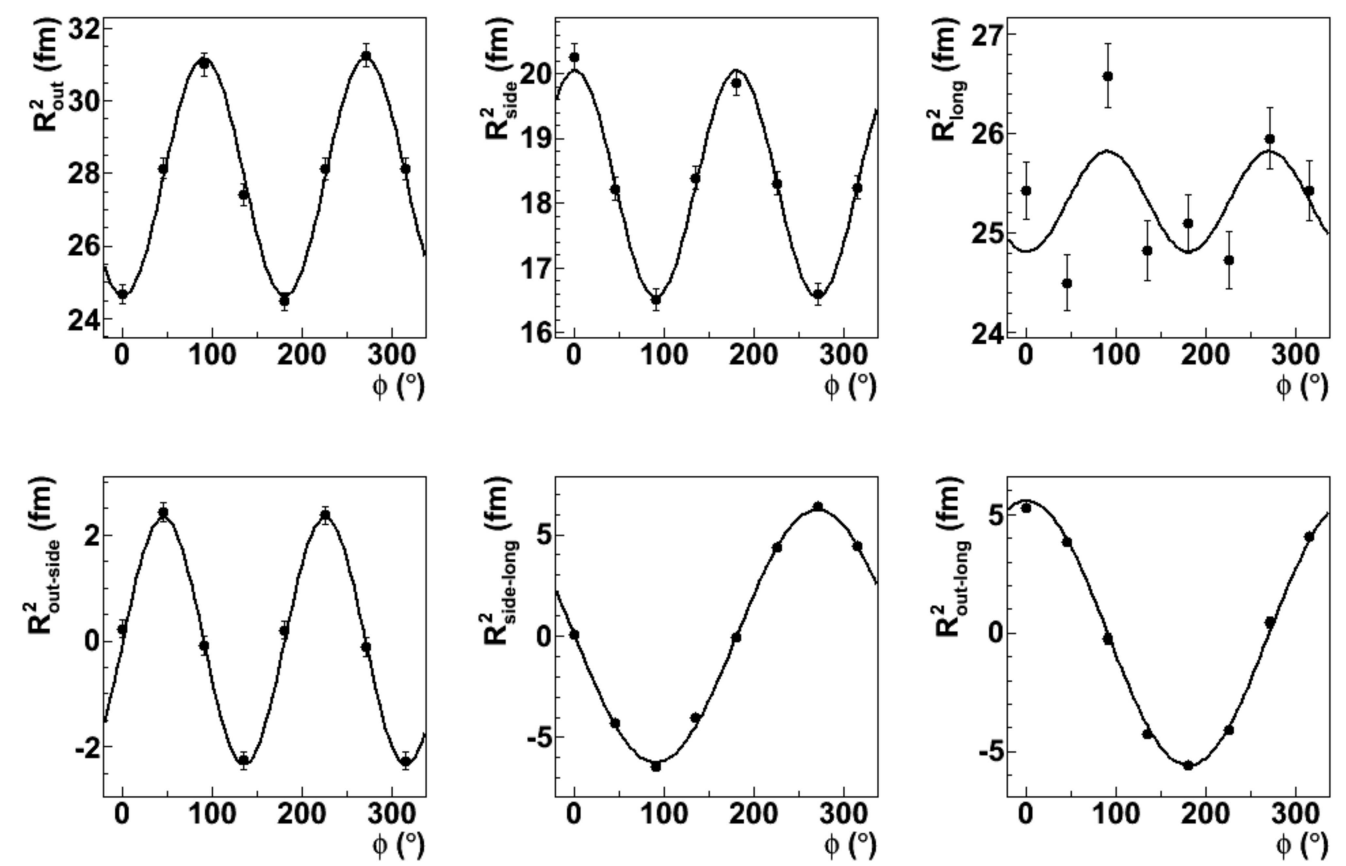

Figure 1: The six HBT radii obtained via fits with Eq. 2.2 to the correlation functions corresponding to different azimuthal angles $\phi$. Curves represent a Fourier decomposition, including terms up to second order. The results are for pions from $\mathrm{Au}+\mathrm{Au}$ collisions at $E_{l a b}=6 \mathrm{~A} \mathrm{GeV}$ with $b=4-8$ fm, $p_{\perp}<0.6 \mathrm{GeV} / \mathrm{c}$ and $|y|<0.6$. Taken from [9].

oscillation is visible in all six radii parameters. From these oscillating radii the Fourier components are extracted using formulas $[7,8]$

$$
\begin{aligned}
R_{s}^{2}(\phi) & =R_{s, 0}^{2}+2 \sum_{n=2,4,6, \ldots} R_{s, n}^{2} \cos (n \phi), \\
R_{o}^{2}(\phi) & =R_{o, 0}^{2}+2 \sum_{n=2,4,6, \ldots} R_{o, n}^{2} \cos (n \phi), \\
R_{o s}^{2}(\phi) & =2 \sum_{n=2,4,6, \ldots} R_{o s, n}^{2} \sin (n \phi), \\
R_{l}^{2}(\phi) & =R_{l, 0}^{2}+2 \sum_{n=2,4,6, \ldots} R_{l, n}^{2} \cos (n \phi), \\
R_{o l}^{2}(\phi) & =2 \sum_{n=1,3,5, \ldots} R_{o l, n}^{2} \cos \phi \\
R_{s l}^{2}(\phi) & =2 \sum_{n=1,3,5, \ldots} R_{s l, n}^{2} \sin \phi .
\end{aligned}
$$

Since the oscillating radii in this analysis are not a continuous function the resulting Fourier coefficients need to be corrected for finite binning effects by applying

$$
\bar{R}_{\mu, n}^{2}=\frac{n \Delta \phi / 2}{\sin n \Delta \phi / 2} R_{\mu, n}^{2}
$$

where $\bar{R}_{\mu, n}$ are the corrected radii of nth-order, and $\Delta \phi$ is the width of the phi bins, in this case $45^{\circ}$.

In Fig. 2 left a projection on the reaction plane of the pion freezeout distribution for $\mathrm{Au}+\mathrm{Au}$ collisions at $E_{l a b}=6 \mathrm{~A} \mathrm{GeV}$ with $b=4-8 \mathrm{fm}, p_{\perp}<0.6 \mathrm{GeV} / \mathrm{c}$ and $|y|<0.6$ is shown. It is obvious that in these non-central collisions the source is not aligned along the beam axis but tilted away from this axis. The black line shows $\theta_{s}$ from a fit to the freezeout distribution with the 

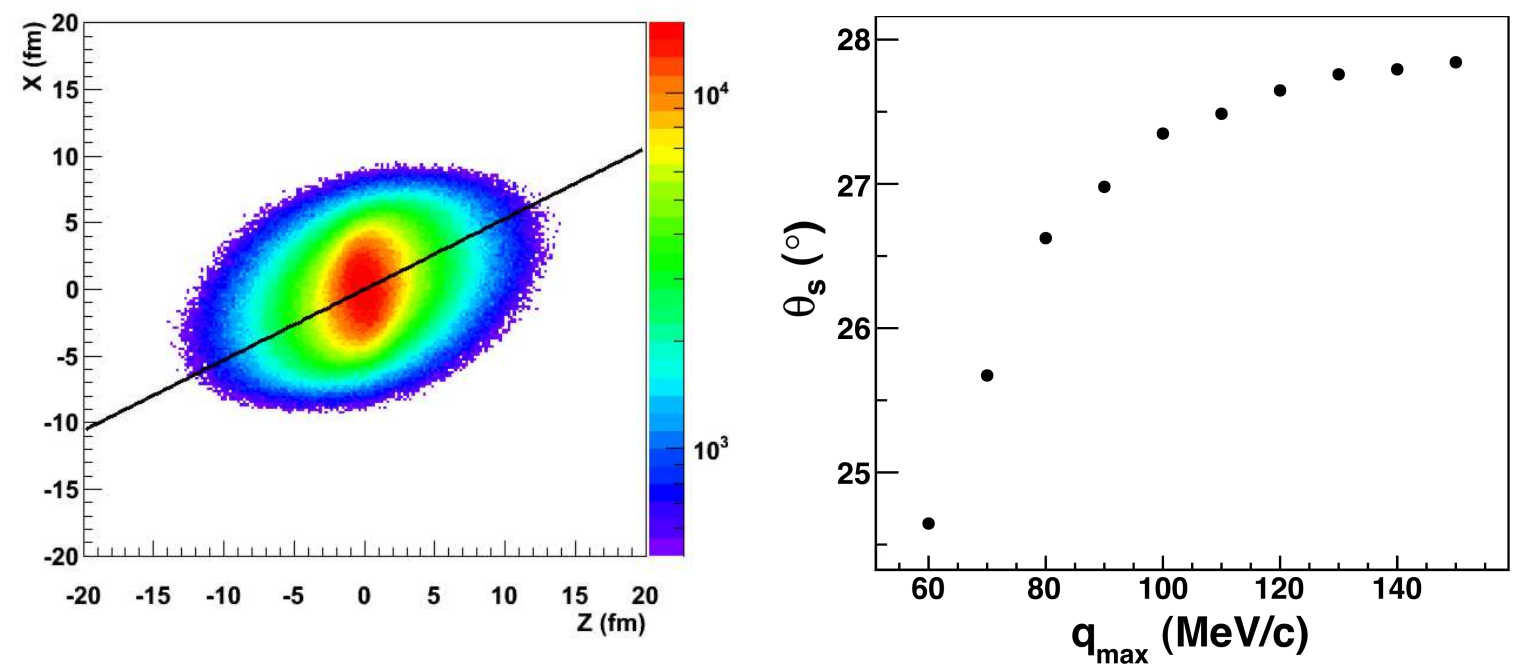

Figure 2: Pion freezeout distribution (left) for $\mathrm{Au}+\mathrm{Au}$ collisions at $E_{\text {lab }}=6 \mathrm{~A} \mathrm{GeV}$ with $b=4-8$ $\mathrm{fm}, p_{\perp}<0.6 \mathrm{GeV} / \mathrm{c}$ and $|y|<0.6$. The black line represents the tilt angle $\theta_{s}$ from the fit to coordinate space. The right side shows $\theta_{s}$ as extracted from the HBT analysis via Eq. 3.4 for different fit ranges to the correlation functions. Taken from [9].

function

$$
f(x, y, z) \sim \exp \left(-\frac{\left(x \cos \theta_{s}-z \sin \theta_{s}\right)^{2}}{2 \sigma_{x^{\prime}}^{2}}-\frac{y^{2}}{2 \sigma_{y}^{2}}-\frac{\left(x \sin \theta_{s}+z \cos \theta_{s}\right)^{2}}{2 \sigma_{z^{\prime}}^{2}}\right) .
$$

Equation 3.3 describes a three dimensional Gaussian ellipsoid tilted away from the beam axis by the angle $\theta_{s}$, where $\sigma_{x^{\prime}}$ and $\sigma_{z^{\prime}}$ are the widths along the principal axes.

Since the freezeout distribution is not experimentally accessible the tilt can not be extracted in this way. However, the tilt angle $\theta_{s}$ is related to the Fourier coefficients of the HBT radii via $[9,10]$

$$
\theta_{s}=\frac{1}{2} \tan ^{-1}\left(\frac{-4 \bar{R}_{s l, 1}^{2}}{R_{l, 0}^{2}-R_{s, 0}^{2}+\bar{R}_{s, 2}^{2}}\right)
$$

In the right plot of Fig. 2 the value of $\theta_{s}$, extracted using the Fourier decomposition of the oscillating HBT radii and using Eq. 3.4, is shown. $\theta_{s}$ is plotted versus the maximal $q$-range $\left(q_{\max }\right)$ used when fitting all the correlation functions. An increase in $\theta_{s}$ with $q_{\max }$ is seen. At the same time a saturation of $\theta_{s}$ shows up at high $q_{\max }$. We interpret this as a sign that different $q$ regions pick up the tilt of different regions in the freezeout distribution. On the left side of Fig. 2 not only a tilt of the source away from the beam axis is seen, but the source is also twisted in itself. However more work needs to be done to clarify the relation between the $q$ dependence of the tilt angle and the changing tilt angles at different points in the freezeout distribution. $\theta_{s}$ is $34^{\circ}-41^{\circ}$ from the fit to freezeout distribution and $27.4^{\circ}-27.9^{\circ}$ from the HBT analysis in the range $100 \mathrm{MeV} / \mathrm{c}$ $<q_{\max }<150 \mathrm{MeV} / \mathrm{c}$. Further investigations showed that the $\theta_{s}$ from the HBT is, as in the present case, always slightly smaller than $\theta_{s}$ extracted from the fit to the freezeout distribution. 
It is natural to extract $\theta_{s}$ also for higher energies, such as the energies at LHC. Further investigations [11] showed however, that $\theta_{s}$ decreases with increasing $\sqrt{s}$ and is basically zero above $\sqrt{s}=56 \mathrm{~A} \mathrm{GeV}$. At higher energies other interesting features can be explored by the HBT technique.

\section{Decoupling time for various system sizes}

At high energies the HBT technique can be used to estimate the decoupling time. For this the hydrodynamically motivated equation $[12,13]$

$$
R_{\text {long }}^{2}=\tau_{f}^{2} \frac{T}{m_{\perp}} \frac{K_{2}\left(m_{\perp} / T\right)}{\left.K_{1} m_{\perp} / T\right)}
$$

is used. Here $\mathrm{T}$ is the pion freezeout temperature, $m_{\perp}=\sqrt{K_{\perp}^{2}+m_{\pi}^{2}}, \tau_{f}$ is the decoupling time and $K_{i}$ are the integer order modified Bessel functions. The decoupling time is extracted by fitting Eq. 4.1 to the $m_{\perp}$ dependence of $R_{\text {long }}$. For all the different systems a temperature of $T=120 \mathrm{MeV}$ is assumed. The explored systems feature $\mathrm{Au}+\mathrm{Au}$ and $\mathrm{Pb}+\mathrm{Pb}$ collisions from $\sqrt{s}=17.3 \mathrm{GeV}$ up to $\sqrt{s}=2.76 \mathrm{TeV}$ for the centralities $0-5 \%, 5-20 \%, 20-40 \%$ and $40-80 \%$, central $\mathrm{C}+\mathrm{C}$ and $\mathrm{Cu}+\mathrm{Cu}$ collisions at $\sqrt{s}=200 \mathrm{GeV}$ and also $\mathrm{p}+\mathrm{p}$ calculations at $\sqrt{s}=7 \mathrm{TeV}$ divided in several multiplicity

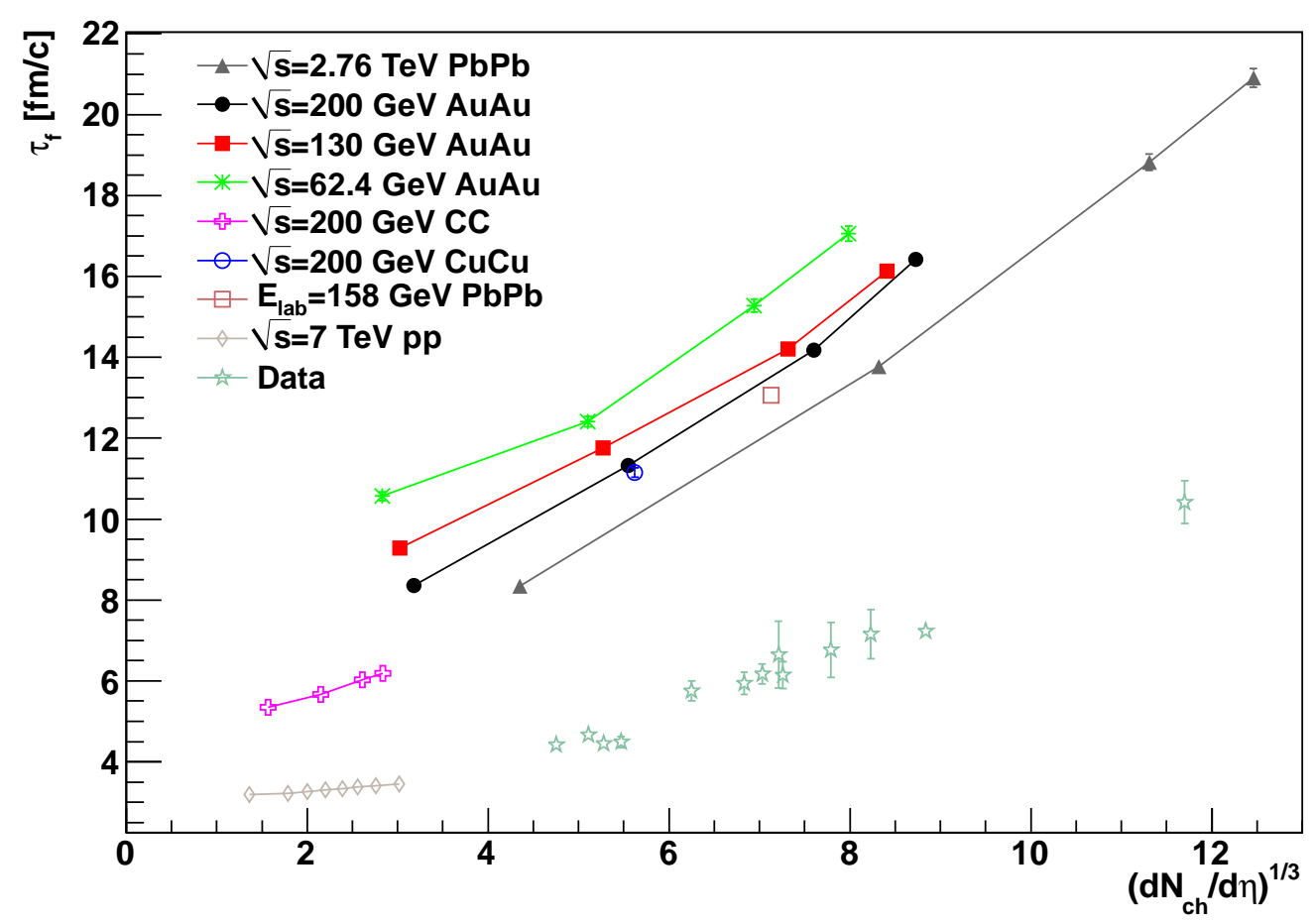

Figure 3: The freezeout time as determined by Eq. 4.1 versus the cube root of the number of charged particles per unit of pseudorapidity. The different points in each energy and system correspond to UrQMD calculations for the centralities $0-5 \%, 5-20 \%, 20-40 \%$ and $40-80 \%$ in the heavy ion case and various multiplicity classes in the proton + proton case. The experimental data(green stars) are from central collisions of gold and lead nuclei at the AGS, SPS, RHIC and LHC [12]. 
classes.

The first interesting feature that shows up in the UrQMD results for the decoupling time is that the scaling with $\left(d N_{c h} / d \eta\right)^{1 / 3}$ is only valid within each energy and nucleus species, but not across energies and species. The second feature is the much smaller decoupling time in $\mathrm{p}+\mathrm{p}$ collisions compared to the decoupling time in $\mathrm{A}+\mathrm{A}$ at the same multiplicities. We relate both observations to the fact that the particle composition and production mechanisms change when changing $\sqrt{s}$. This alters the decoupling time of the system. The decoupling time from UrQMD is overestimated compared to the experimental data. We attribute this to the well known fact that the pressure in the early stage is not strong enough in the cascade mode calculations. A higher pressure would lead to a more explosive expansion of the system, thus leading to smaller regions of homogeneity and a faster decoupling $[14,15]$.

\section{Summary}

We showed that in UrQMD the shape of the freezeout region of non-central heavy ion collisions is tilted away from the beam axis. The angle of this tilt was extracted via azimuthal sensitive HBT and via a direct fit to the freezeout distribution. It was found that the tilt angle from a fit to the freezeout distribution is always slightly bigger than from the HBT analysis, but still close by. With increasing energy the tilt angle decreases. At high energy we used HBT to estimate the decoupling time of various system and energies. We found that the decoupling time scales with $\left(d N_{c h} / d \eta\right)^{1 / 3}$ within each energy but the scaling is broken across energies.

\section{References}

[1] S. A. Bass et al., Prog. Part. Nucl. Phys. 41, 255-369 (1998).

[2] M. Bleicher et al., J. Phys. G G25, 1859-1896 (1999).

[3] H. Petersen, M. Bleicher, S. A. Bass, H. Stocker, [arXiv:0805.0567 [hep-ph]].

[4] H. Petersen, J. Steinheimer, G. Burau, M. Bleicher, H. Stocker, Phys. Rev. C78, 044901 (2008).

[5] Download the most recent UrQMD source code from http://urqmd.org/

[6] U. A. Wiedemann and U. W. Heinz, Phys. Rept. 319, 145 (1999).

[7] U. W. Heinz, A. Hummel, M. A. Lisa and U. A. Wiedemann, Phys. Rev. C 66, 044903 (2002).

[8] F. Retiere and M. A. Lisa, Phys. Rev. C 70, 044907 (2004).

[9] E. Mount, G. Graef, M. Mitrovski, M. Bleicher and M. A. Lisa, Phys. Rev. C 84, 014908 (2011).

[10] M. A. Lisa, U. W. Heinz and U. A. Wiedemann, Phys. Lett. B 489, 287 (2000).

[11] M. A. Lisa, E. Frodermann, G. Graef, M. Mitrovski, E. Mount, H. Petersen and M. Bleicher, New J. Phys. 13, 065006 (2011).

[12] K. Aamodt et al. [ALICE Collaboration], Phys. Lett. B 696, 328 (2011).

[13] A. N. Makhlin, Y. .M. Sinyukov, Z. Phys. C39, 69 (1988).

[14] S. Pratt, Acta Phys. Polon. B 40, 1249 (2009).

[15] Q. Li, M. Bleicher and H. Stocker, Phys. Lett. B 659, 525 (2008). 